\title{
Associação da postura craniocervical com disfunção temporomandibular e oclusão dentária
}

\author{
Association of craniocervical posture with temporomandibular dysfunction and dental \\ occlusion
}

\author{
Paulo Raimundo Rosário Lopes ${ }^{1 *}$, Gabriela Botelho Martins², Roberto Paulo Correia de Araújo³ \\ ${ }^{1}$ Doutor pelo Programa de Pós Graduação em Processos Interativos dos órgãos e Sistemas do Instituto de \\ Ciências da Saúde da Universidade Federal da Bahia; ${ }^{2}$ Professora Associado do Instituto de Ciências da Saúde da \\ Universidade Federal da Bahia. Doutora em Estomatologia Clínica pela PUC-RS; ${ }^{3}$ Professor Titular do Instituto de \\ Ciências da Saúde da Universidade Federal da Bahia. Livre-docência em Bioquímica Oral pela UGF.
}

\begin{abstract}
Resumo
Não há consenso na literatura quanto à associação entre postura craniocervical (PCC), disfunção temporomandibular (DTM) e oclusão dentária. Ainda assim, admite-se que a articulação temporomandibular está intimamente envolvida com a biomecânica cervical e cintura escapular. Objetivo: traçar o perfil das associações entre PCC, DTM e oclusão dentária. Metodologia: estudo observacional em corte transversal realizado em universitários, de ambos os sexos, com idade entre 18 e 40 anos. Os voluntários foram avaliados pelo RDC/TMD, biofotogrametria, sendo esta realizada através do Software de Avaliação Postural (SAPO) pelos ângulos A1(tragushorizontal), A2 (tragus-C7-horizontal), A3 (tragus - acrômio-vertical) e distância tóraco-cervical, além da análise quanto ao tipo de oclusão dentária. $\mathrm{O}$ tratamento dos dados foi feito a partir de tendência central, razão de prevalência e análise de correspondência múltipla assimétrica. Resultados: foram avaliados 88 voluntários com mediana da idade de 22 anos, sendo 27 do sexo masculino e 62 do feminino. A amostra foi dividida em quatro grupos, de acordo com a presença de DTM, padrão de dor à palpação, cefaléia, PCC e oclusão. As maiores associações observadas foram: DTM tipo II com a distoclusão tipo dois, DTM tipo I com flexão de cabeça e retificação da coluna cervical; mesioclusão com alinhamento da coluna cervical e indivíduos sem dor com anteriorização da cabeça e distoclusão tipo um. Conclusão: este estudo demonstrou que a DTM não interfere na postura da cabeça, mas que a má oclusão pode alterar a postura craniocervical nos planos sagital e frontal além de aumentar a lordose cervical.

Palavras-chaves: Postura. Maloclusão. Síndrome da Disfunção da Articulação Temporomandibular.
\end{abstract}

\begin{abstract}
There is no consensus in the literature about the association between craniocervical posture (CCP), temporomandibular dysfunction (TMD) and dental occlusion, although it is admitted that the temporomandibular joint is closely involved with the cervical and shoulder girdle biomechanics. Objective: to identify the profile about associations between CCP, TMJ and dental occlusion. Methodology: observational cross-sectional study, conducted on both sexes college student aged 18 to 40 years. The subjects were evaluated by RDC/TMD, biophotogrammetry, using Postural Assessment Software (SAPO), according to A1 (tragus-horizontal), A2 (tragus-C7horizontal), A3 (tragus-acromion-vertical) and cervical thoracic distance, besides the analysis of the type of dental occlusion. Data analysis was performed by measures of central tendency, prevalence ratio and asymmetric multiple correspondence. Results: a total of 88 volunteers with a median age of 22 years were evaluated, 27 male and 62 female. The profiles was divided into four groups according to the presence of TMD, pain on palpation, headache, CCP and occlusion. The associations observed were: type II TMD with type two distocclusion, type I TMD with head flexion and cervical spine rectification; mesiocclusion with cervical spine alignment and pain-free individuals with anteriorization of the head and distocclusion type one. Conclusão: this study demonstrated that TMD does not interfere with the head position, but that malocclusion may alter craniocervical posture in the sagittal and frontal planes and increase cervical lordosis.
\end{abstract}

Keywords: Posture. Malocclusion. Temporomandibular Joint Dysfunction Syndrome.

\section{INTRODUÇÃO}

A Articulação Temporomandibular (ATM), tem sido apontada como uma das mais solicitadas do corpo humano, estando relacionada com a coluna cervical e a cintura

Correspondente/Corresponding: *Paulo Raimundo Rosário Lopes Instituto de Ciências da Saúde (UFBA), Programa de Pós-graduação em Processos Interativos dos Órgãos e Sistemas - End: Av. Reitor Miguel Calmon, s/n. Sala 410. Vale do Canela. Salvador/BA. CEP: 40. 231-300.

- Tel: (71) 98808-7150 - E-mail: paulo.rrl@gmail.com escapular através de um sistema neuromuscular comum (MONTEIRO et al., 2013). As Disfunções temporomandibulares (DTM) são condições de etiologia multifatorial que envolve alterações da estrutura e função do sistema mastigatório, ATM e músculos da cabeça e pescoço, caracterizada principalmente por: dor, ruídos articulares, travamento, limitação de movimento, sensibilidade muscular, além de função mandibular irregular ou com desvio (RIES; BERZIN, 2008). 
A associação entre postura de cabeça, postura cervical, posição mandibular e DTM tem sido amplamente pesquisada e ainda é motivo de divergências. Enquanto alguns autores afirmam que a lordose cervical aumenta com as alterações oclusais (CASTILLO et al., 2016) outros afirmam que desequilíbrios posturais craniocervicais ocorrem em indivíduos independente das condições estomatognáticas (ROCHA et al., 2017). A maloclusão dentária, caracterizada por desadaptação no posicionamento das arcadas, parece alterar a postura da cabeça no plano sagital, assim como no plano frontal e transverso (BIASOTTO-GONZALEZ et al., 2008) no entanto, autores afirmam que a maloclusão não se apresenta como um fator desencadeador ou interfere na severidade DTM (LEMOS et al, 2015)

Apesar de dados clínicos apontarem para o fato de que $80 \%$ das etiologias das DTM estejam associadas às más posturas, apenas $15 \%$ destas estão propriamente relacionadas (NOTA et al., 2017). Os desvios posturais, como anteriorização da cabeça, retificação da coluna cervical e assimetria de ombros, influenciam no surgimento das DTM's bem como nos consequentes sintomas dolorosos. Alguns sinais e sintomas cervicais podem estar associados à severidade da DTM, entretanto não se considera o inverso verdadeiro, visto que a sintomatologia cervical pode ser melhor definida como um fator perpetuante da disfunção (MONTEIRO et al., 2013). Essa alteração postural de cabeça pode estar relacionada ainda com a redução da amplitude de movimento cervical e com dores musculares (CASTILLO et al., 2016).

Dentro da área de motricidade orofacial, muitos profissionais negligenciam as associações entre músculos da face, da cabeça e relacionados à posturologia, o que interfere negativamente nas propostas terapêuticas e resultados funcionais do sistema estomatognático. Uma melhor compreensão dessas condições contribuiria, portanto, para abordagens terapêuticas mais abrangentes, coerentes e, consequentemente, mais efetivas. Dessa forma, o objetivo do presente estudo foi verificar a associação entre a postura craniocervical com DTM e oclusão dentária em estudantes universitários.

\section{METODOLOGIA}

Realizou-se um estudo observacional, transversal com amostra não probabilística, constituída por 125 voluntários adultos, estudantes do Instituto de Ciências da Saúde e Faculdade de Odontologia da Universidade Federal da Bahia (UFBA), e Centro Universitário Jorge Amado, Salvador, Ba. O projeto foi aprovado pelo Comitê de Ética e Pesquisa da Fundação de Fomento à Tecnologia e à Ciência das Faculdades de Tecnologia e Ciências CEP/ IMES, sob protocolo no 3841, respeitando a resolução 466/12 do CNS/MS para pesquisa em seres humanos.

Foram incluídos indivíduos devidamente matriculados nas instituições de ensino, com 18 a 40 anos de ambos os sexos, que se voluntariassem e aceitassem participar do estudo mediante assinatura do Termo de Consentimento Livre e Esclarecido. Foram excluídos estudantes com diagnóstico de doenças reumáticas ou neurológicas, que faziam uso de placa oclusal, gestantes ou aqueles que estivessem realizando intervenção terapêutica para DTM ou postura nos últimos trinta dias anteriores ao estudo, como: tratamento fisioterapêutico, uso de antidepressivos, miorrelaxantes e antiinflamatórios.

Os voluntários preencheram um questionário, sob supervisão de um Fisioterapeuta, contendo dados pessoais e os Critérios Diagnósticos para Pesquisa em Desordens Temporomandibulares (RDC/TMD). A avaliação da oclusão dentária foi realizada por um profissional cirurgião-dentista com base na classificação de oclusão funcional de Angle (ANGLE, 1899) definidas como: Classe I (neutrocluão), Classe lla (distoclusão divisão 1 incisívos projetados), Classe Ilb (distoclusão divisão 1 incisívos verticais ou retroinclinados) e Classe III (mesioclusão). A avaliação postural foi realizada por um fisioterapeuta pela biofotogrametria a partir dos planos frontal e sagital. A câmera fotográfica (Samsung ST500) foi travada a 90응 da posição horizontal, a uma distância de $3 \mathrm{~m}$ da parede e altura relativa à metade da altura do voluntário com foco centralizado. Para padronização das fotos, o indivíduo deveria estar com os pés paralelos, olhar na linha do horizonte e realizar uma inspiração profunda em três tempos; e a mesma base de sustentação foi garantida com a marcação do pé do voluntário em um tapete de EVA preto.

As imagens foram avaliadas pelo software SAPO (Figura 1). Com o ângulo 1 ( $A 1$ - ângulo entre tragus direito/ esquerdo e a horizontal), verificou-se a inclinação/rotação da cabeça no plano frontal; com o ângulo 2 (A2 ângulo entre tragus, C7 e a horizontal), verificou-se o alinhamento horizontal da cabeça em perfil, caracterizando flexão ou extensão da cabeça (RAINE; TWOMEY, 1997); com o ângulo 3(A3 ângulo entre acrômio, tragus e a vertical) verificou-se o do alinhamento vertical da cabeça; e com a a distância toraco-cervivcal (DTC/A3), e grau de lordose cervical pela linha que tangenciava o ápice da curvatura torácica e o ápice da curvatura cervical (BOLZAN et al., 2011). 
Figura 1 - Ângulos avaliados pelo SAPO. [ângulo entre tragus direito/esquerdo e a horizontal (A1); ângulo entre tragus, C7 e a horizontal (A2); ângulo entre acrômio, tragus e a vertical (A3); distância linha torácica e ápice da concavidade cervical (DTC)].

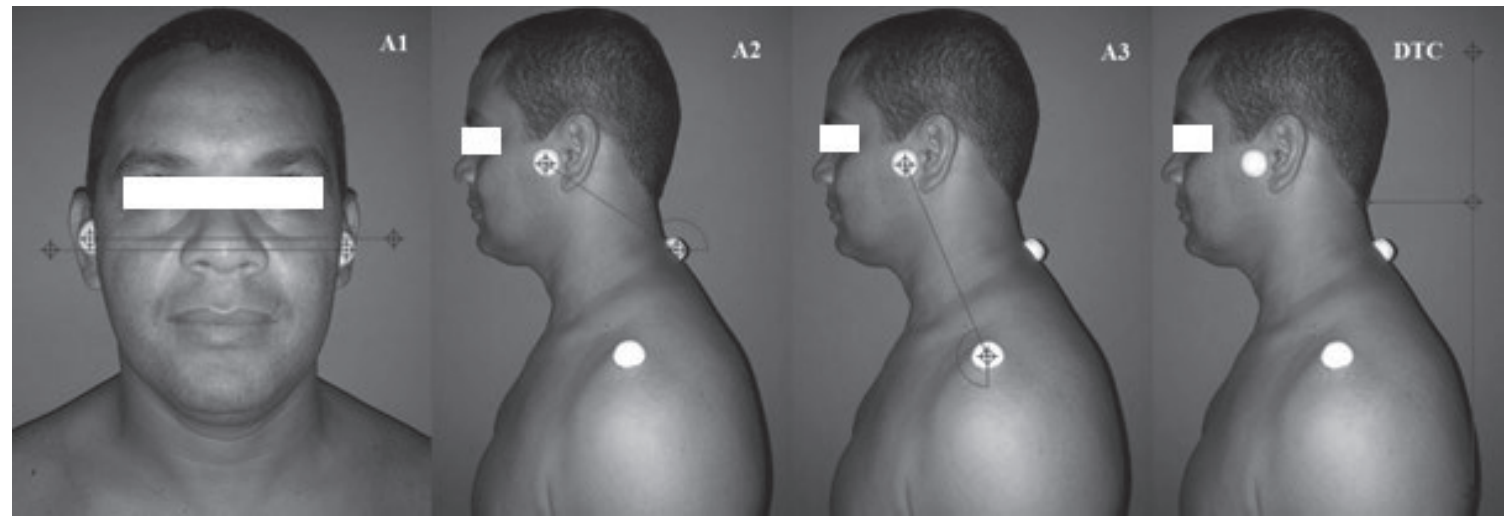

Fonte: Elaboração própria (2019).

Os dados quantitativos foram avaliados mediante estatística descritiva com seus respectivos índices de dispersão. Para obtenção do perfil da população do estudo, foi realizada a análise multivariada e, pelo contexto multifatorial, foi efetuada análise de correspondência múltipla assimétrica, com a decomposição de autovalores da matriz de Burt, ajustada pelas inércias e com as coordenadas padronizadas multiplicadas pelas massas, para a avaliação das relações geométricas em distâncias qui-quadrado do contingenciamento das variáveis estudadas em um contexto multidimensional (GREENACRE, 2007).

Para a obtenção direta das razões de prevalência, ajustadas quando necessário, foi realizado modelo linear generalizado via regressão log-binomial, binomial negativa ou Poisson. As variáveis incluídas no modelo foram selecionadas a partir da inspeção visual do mapa de correspondência, e a sua manutenção no modelo se deu a partir de um ponto de corte arbitrário maior do que $30 \%$ nas razões de prevalência ajustadas. Devido ao plano amostral não probabilístico, não foi necessário o cálculo de estatísticas inferenciais para a decisão da manutenção de variáveis no modelo final (BARROS; HIRAKATA, 2003). $A$ análise estatística foi realizada no pacote estatístico $R$ para Ubuntu.

\section{RESULTADOS}

Oitenta e oito voluntários concluíram o estudo, sendo 26 homens e 62 mulheres com. A mediana da idade foi de 22 anos com mínimo de 18 e máximo de 39 anos. A tabela 1 apresenta o perfil da amostra de acordo com presença ou não de DTM, sinais e sintomas mais frequentes, oclusão dentária e postura craniocervical.
Tabela 1 - Perfil das variáveis em relação à população $(n=88)$

\begin{tabular}{|c|c|c|c|}
\hline VARIÁVEIS & MASCULINO & FEMININO & TOTAL \\
\hline Voluntários & 26 & 62 & 88 \\
\hline Sem DTM & $16(39 \%)$ & $25(61 \%)$ & $41(46,6 \%)$ \\
\hline Com DTM: & $10(21,3 \%)$ & $37(78,7 \%)$ & $47(53,4 \%)$ \\
\hline la & $2(7,7 \%)$ & $11(17,7 \%)$ & 13 \\
\hline $\mathrm{lb}$ & $0(-)$ & $8(12,9 \%)$ & 8 \\
\hline lia & $1(3,8 \%)$ & $7(11,3 \%)$ & 8 \\
\hline lib & $0(-)$ & $1(1,6 \%)$ & 1 \\
\hline lic & $1(3,8 \%)$ & $0(-)$ & 1 \\
\hline IIla & $11(42,3 \%)$ & $23(37,1 \%)$ & 34 \\
\hline IIIb & $1(3,8 \%)$ & $2(3,2 \%)$ & 3 \\
\hline IIIc & 0 & $2(3,2 \%)$ & 2 \\
\hline $\begin{array}{l}\text { № de sítios de dor à } \\
\text { palpação articular }\end{array}$ & $21(30,4 \%)$ & $48(69,6 \%)$ & $69(78,4 \%)$ \\
\hline $\begin{array}{l}\text { № de sítios de dor à } \\
\text { palpação muscular }\end{array}$ & $26(30,2 \%)$ & $60(69,8 \%)$ & $86(97,7 \%)$ \\
\hline $\begin{array}{l}\text { Queixa Cefaleia/ } \\
\text { Enxaqueca }\end{array}$ & $4(15,4 \%)$ & $36(58 \%)$ & $40(45,4 \%)$ \\
\hline $\begin{array}{l}\text { Hábitos deletérios } \\
\text { orofaciais }\end{array}$ & $15(57,7 \%)$ & $23(37,1 \%)$ & $38(43,1 \%)$ \\
\hline \multicolumn{4}{|l|}{ Oclusão dentária: } \\
\hline Normo-oclusão & $23(88,5 \%)$ & $22(35,5 \%)$ & $45(51,1 \%)$ \\
\hline Disto-oclusão tipo a & $3(11,5 \%)$ & $23(37 \%)$ & $26(29,5 \%)$ \\
\hline Disto-oclusão tipo b & 0 & $9(14,5 \%)$ & $9(10,2 \%)$ \\
\hline Mésio-oclusão & $1(3,8 \%)$ & $7(11,3 \%)$ & $8(9 \%)$ \\
\hline
\end{tabular}

Alterações posturais entre ângulos e DTC:

A1:

\begin{tabular}{lrrr} 
Alinhamento & $5(19,2 \%)$ & $10(16,1 \%)$ & $15(17 \%)$ \\
Inclinação á direita & $10(38,5 \%)$ & $28(45,1 \%)$ & $38(43,1 \%)$ \\
$\begin{array}{l}\text { Inclinação à } \\
\text { esquerda }\end{array}$ & $11(42,3 \%)$ & $24(38,7 \%)$ & $35(39,8 \%)$ \\
A2: & & & \\
Flexão & $7(26,9 \%)$ & $37(59,7 \%)$ & $44(50 \%)$ \\
Extensão & $19(73 \%)$ & $25(40,3)$ & $44(50 \%)$ \\
A3: & & & \\
Alinhamento & $10(38,5 \%)$ & $25(40,3 \%)$ & $35(39,8 \%)$ \\
Anteriorização & $16(61,5 \%)$ & $37(59,7 \%)$ & $53(60,2 \%)$ \\
DTC: & & & \\
Alinhamento & $1(3,8 \%)$ & $25(40,3 \%)$ & $26(29,5 \%)$ \\
Retificação & $8(30,8 \%)$ & $30(48,4 \%)$ & $38(43,2 \%)$ \\
Anteriorização & $17(65,4 \%)$ & $7(11,3 \%)$ & $24(27,3 \%)$ \\
\hline
\end{tabular}

Fonte: Elaboração própria (2019). 
Na figura 2, observam-se os perfis de associação entre as variáveis estudadas. O primeiro quadrante é composto de indivíduos sem DTM, com padrão de dor à palpação leve, cabeça anteriorizada ou inclinada à direita. O segundo quadrante apresenta indivíduos do sexo masculino, acima de 22 anos, sem DTM, com normoclusão (classe I) e postura craniocervical de extensão da cabeça com anteriorização da coluna cervical.

Compõem o terceiro quadrante os indivíduos com DTM subgrupos II (deslocamento discais) e III (alterações articulares), associados ao padrão de dor moderado ou severo, com provável inclinação à esquerda e classe Ilb de Angle. Compõem o quarto quadrante os voluntários do sexo feminino, com faixa etária até 22 anos, com DTM do tipo I (miogênica), que tinham cefaleia/enxaqueca nos últimos seis meses, mantinham postura craniocervical em flexão de cabeça com retificação da coluna cervical e classe de oclusão Ila ou III.

Quanto à proximidade dos pontos no gráfico, referente às áreas demarcadas, foi possível observar que: DTM tipo I está mais associada à postura craniocervical em flexão de cabeça com retificação da coluna cervical; indivíduos com mésio-oclusão apresentam alinhamento da coluna cervical; e indivíduos com anteriorização não apresentam dor à palpação e podem ter maloclusão tipo lla.

Figura 2 - Análise multivariada da população. (sx1 = sexo masculino, sx2 = sexo feminino, $f \times 0=$ faixa etária até 22 anos, fx2 = faixa etária acima dos 22 anos, $p 190=$ sensação de somente pressão dos dedos, $p 191=$ padrão de dor à palpação leve, p192 = padrão de dor à palpação moderada, $p 193=$ padrão de dor à palpação severa, ho= sem cefaleia/enxaqueca, $h 1==$ com cefaleia/enxaqueca, o0 = normo-oclusão, o1 = disto-oclusão a, o2 = disto-oclusão b, o3 = mésio-oclusão; $A 11=$ alinhamento $A 1, A 12=$ inclinação à direita, A13 = inclinação à esquerda, A21 = extensão da cabeça, $A 22$ = flexão da cabeça, $A 31=$ alinhamento A3, A32 = anteriorização A3, DTC1 = retificação cervical, DTC2 = alinhamento cervical, DTC3 = anteriorização cervical, dtmIO = sem DTM subgrupo miogênica, $d t m / 1=$ com DTM subgrupo miogênica, $d t m l l O=$ sem DTM subgrupo deslocamento do disco, dtmll1 = com DTM subgrupo deslocamento do disco, dtmIIIO = sem DTM subgrupo articular, dtmIII1 = com DTM subgrupo articular).

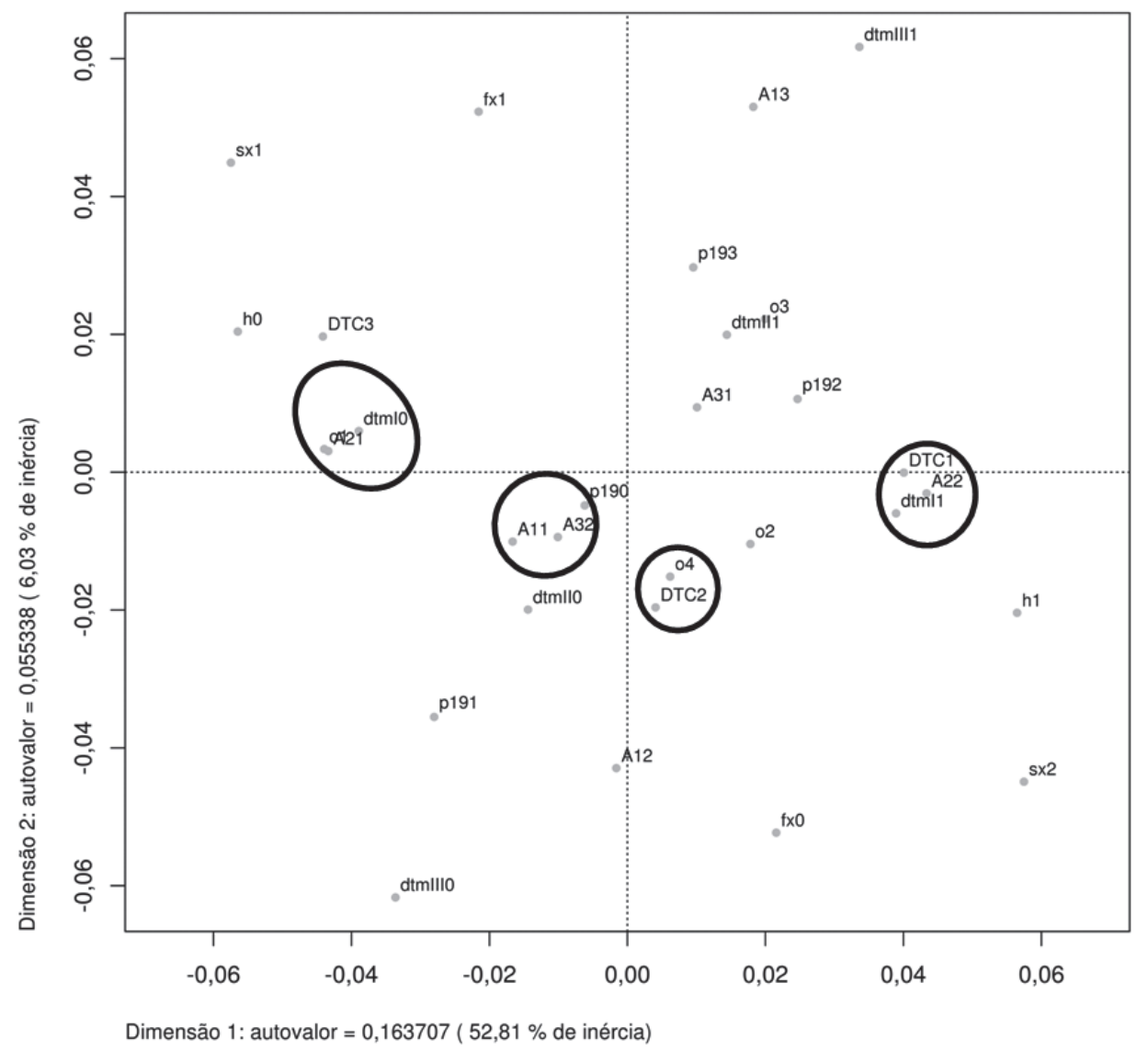

Fonte: Elaboração própria (2019).

As associações entre presença ou não de DTM, demais variáveis do RDC/TMD e postura craniocervical encontram-se na tabela 2 , bem como a associação entre classe de oclusão dentária e postura craniocervical estão descritas na tabela 3. 
Tabela 2 - Associação entre DTM e demais variáveis na população

\begin{tabular}{|c|c|c|c|}
\hline VARIÁVEL & Com DTM & Sem DTM & TOTAL \\
\hline \multicolumn{4}{|l|}{ Dor muscular: } \\
\hline Sim & $46(53,5 \%)$ & $40(46,5 \%)$ & 86 \\
\hline Não & $1(50 \%)$ & $1(505)$ & 2 \\
\hline \multicolumn{4}{|l|}{$\mathrm{RP}=1,15$} \\
\hline \multicolumn{4}{|l|}{ Dor articular: } \\
\hline Sim & $42(61,8 \%)$ & $26(38,2 \%)$ & 68 \\
\hline \multirow[t]{2}{*}{ Não } & $5(26,3 \%)$ & $14(73,7 \%)$ & 19 \\
\hline & & & $R P: 1,61$ \\
\hline \multicolumn{4}{|l|}{ Cefaleia/Enxaqueca: } \\
\hline Sim & $27(69,3 \%)$ & $12(30,7 \%)$ & 39 \\
\hline \multirow[t]{2}{*}{ Não } & $20(40,8 \%)$ & $29(59,2 \%)$ & 49 \\
\hline & & & $R P=1,7$ \\
\hline \multicolumn{4}{|l|}{ Hábitos deletérios orofaciais } \\
\hline $\operatorname{Sim}$ & $22(59,4 \%)$ & $15(40,5 \%)$ & 37 \\
\hline \multirow[t]{2}{*}{ Não } & $24(49 \%)$ & $25(51 \%)$ & 49 \\
\hline & & & $R P=1,7$ \\
\hline \multicolumn{4}{|l|}{ A1 (ângulo tragus-horizontal): } \\
\hline Alinhamento & $4(26,7 \%)$ & $11(73,3 \%)$ & 15 \\
\hline Inclinação/rotação direita & $21(55,3 \%)$ & $17(44,7 \%)$ & 38 \\
\hline \multirow[t]{2}{*}{ Inclinação/rotação esquerda } & $22(62,8 \%)$ & $13(37,2 \%)$ & 35 \\
\hline & \multicolumn{3}{|c|}{$R P$ : incl/rot direita $=2,07 / /$ incl/rot esquerda $=2,35$} \\
\hline \multicolumn{4}{|l|}{ A2 (ângulo tragus-C7-horizontal): } \\
\hline Flexão & $19(44,2 \%)$ & $24(55,8 \%)$ & 43 \\
\hline Extensão & $28(62,2 \%)$ & $17(37,8 \%)$ & 45 \\
\hline \multicolumn{4}{|c|}{ A3 (ângulos tragus-acrômio-vertical): } \\
\hline Alinhada & $20(57,2 \%)$ & $15(42,8 \%)$ & 35 \\
\hline \multirow[t]{2}{*}{ Anteriorização } & $27(50,9 \%)$ & $26(49,1 \%)$ & 53 \\
\hline & & & $R P=0,9$ \\
\hline \multicolumn{4}{|l|}{ Distância tóraco cervical: } \\
\hline Alinhamento & $20(64,5 \%)$ & $11(35,9 \%)$ & 31 \\
\hline Retificação & $18(55,5 \%)$ & $15(45,5 \%)$ & 33 \\
\hline \multirow[t]{2}{*}{ Anteriorização } & $9(37,55)$ & $15(62,5 \%)$ & 24 \\
\hline & \multicolumn{3}{|c|}{ RP: Retificação $=0,86 / /$ Anteriorização $=0,58$} \\
\hline
\end{tabular}

Fonte: Elaboração própria (2019).

Tabela 3 - Associação do tipo de oclusão e condições posturais na população do estudo

\begin{tabular}{|c|c|c|c|c|c|}
\hline \multirow[b]{2}{*}{ Variáveis posturais } & \multirow[b]{2}{*}{$\begin{array}{l}\text { Normo- } \\
\text { oclusão }\end{array}$} & \multirow[b]{2}{*}{$\begin{array}{r}\text { Disto-oclusão } \\
\text { tipo } 1 \\
\end{array}$} & \multicolumn{2}{|c|}{ TIPO DE OCLUSÃO DENTÁRIA } & \multirow[b]{2}{*}{ Total } \\
\hline & & & $\begin{array}{r}\text { Disto-oclusão } \\
\text { tipo } 2\end{array}$ & $\begin{array}{l}\text { Mésio- } \\
\text { oclusão }\end{array}$ & \\
\hline \multicolumn{6}{|l|}{ A2 } \\
\hline Flexão & $20(44,4 \%)$ & $11(42,3 \%)$ & $7(77,8 \%)$ & $6(75 \%)$ & 44 \\
\hline \multirow[t]{2}{*}{ Extensão } & $25(55,6 \%)$ & $15(57,7 \%)$ & $2(22,2 \%)$ & $2(25 \%)$ & 44 \\
\hline & & \multicolumn{4}{|c|}{$R P: \| a=1,03$ ext e 1,45 flex $/ \| b=1,6$ ext e 1,75 flex $/ I I=1,55$ ext e 1,68 flex } \\
\hline \multicolumn{6}{|l|}{ A3: } \\
\hline Alinhamento & $22(48,9 \%)$ & $7(26,9 \%)$ & $3(33,3 \%)$ & $3(37,5 \%)$ & 35 \\
\hline \multirow[t]{2}{*}{ Anteriorização } & $23(51,1 \%)$ & $19(73,1 \%)$ & $6(66,7 \%)$ & $5(62,5 \%)$ & 53 \\
\hline & & & \multicolumn{3}{|c|}{$R P:\|a=1,43 /\| b=1,30 / I I I=1,2$} \\
\hline \multicolumn{6}{|l|}{ Distância torracocervical: } \\
\hline Alinhamento & $15(33,3 \%)$ & $9(34,6 \%)$ & $6(66,7 \%)$ & $3(37,5 \%)$ & 33 \\
\hline Retificação & $12(26,7 \%)$ & $13(50 \%)$ & $3(33,3 \%)$ & $3(37,5 \%)$ & 31 \\
\hline \multirow[t]{2}{*}{ Anteriorização } & $18(40 \%)$ & $4(15,4 \%)$ & $0(0 \%)$ & $2(25 \%)$ & 24 \\
\hline & & & \multicolumn{3}{|c|}{$R P: \| a=1,53$ ant e $1,5 \%$ ret $/ I I I=1,25$ ant e 1,12 re } \\
\hline
\end{tabular}

Fonte: Elaboração própria (2012). 


\section{DISCUSSÃO}

A prevalência do sexo feminino pode ser justificada pela maior quantidade de mulheres nas instituições participantes, além de serem mais atentas e interessadas aos cuidados com o próprio corpo (COLARES; FRANCA; GONZALEZ, 2009). Tal estatística se mantém no quesito presença de DTM, numa proporção de 3:1 entre os sexos. Estes achados estão de acordo com a literatura, que afirma serem as mulheres mais propensas à DTM numa proporção entre 2:1 a 5:1 (FRANCO et al., 2011; MANFREDINI et al., 2011). Entre os fatores mais aceitos para este fenômeno, estão condições fisiológicas como: maior lassidão ligamentar, que reduz a estabilidade da ATM, e também condições hormonais, que as tornariam mais propensas a tensões físicas e psíquicas (GIANNAKOPOULOS et al., 2010). Autores relatam ainda que a maior quantidade de receptores para estrogênio na ATM pode ser responsável por esta alta prevalência (LOPES; CAMPOS; NASCIMENTO, 2011).

A frequência de DTM em 53,4\% dos estudantes avaliados está de acordo com estudo epidemiológico que afirma que tal condição pode chegar à faixa de $1 \%$ a $75 \%$ para dor orofacial, dor cervical, cefaleia e enxaqueca (GONÇALVES et al., 2010). No entanto, quando se analisa os subgrupos de DTM, os diagnósticos mais prevalecentes foram do subgrupo la, II e III, com predomínio do III, diferindo dos achados de Manfredini et al. (2011) que mostrou, em uma meta-análise, prevalência global de $45,3 \%$ para o subgrupo I, $41,1 \%$ para o subgrupo II e $30,1 \%$ para o subgrupo III.

Esta diferença pode ser justificada pelos hábitos deletérios orofaciais (HDO) que conferem à articulação uma predisposição a desgastes. Apesar da baixa prevalência em jovens, atualmente, admite-se que o pico de idade para DTM varia com o tipo da desordem. Indivíduos até 38 anos têm prevalência igual para as três classificações de DTM, enquanto indivíduos acima de 39 anos têm maior prevalência para os subgrupos I e III (GUARDA-NARDINI, 2012).

Com base no RDC/TMD, a avaliação dos HDO estão relacionados ao ato de ranger os dentes durante o sono ou dia, melhor definido por bruxismo do sono e da vigília. Pôde-se observar que 38 (43,1\%) voluntários apresentavam este sinal, e 32 destes relatavam DTM. Dos que apresentaram a disfunção, a do tipo I (miogênica) foi observada em $15 \%$ destes indivíduos, concordando com a literatura (BRANCO et al., 2008). Ainda assim atualmente as evidências afirmam que não há e não se pode traçar uma relação entre bruxismo e DTM nem mesmo como fator perpetuante (MANFREDINI; LOBEZOO, 2010)

A dor à palpação foi encontrada em $97,72 \%$ da amostra estudada durante o exame físico do RDC/TMD, concordando ser este sintoma o que mais prevalece em jovens acima dos 16 anos do sexo feminino (KARIBE et al, 2012). A razão de prevalência para dor muscular e articular à palpação foi de $15 \%$ e $61 \%$ respectivamente, identificando uma alta probabilidade destes sintomas em indivíduos com DTM em relação aos sem DTM neste estudo. Dados estes apoiados por estudos anteriores que afirmam que a dor, é mais prevalente em estudantes das áreas de saúde e ciências e que, quanto maior a severidade da DTM, o risco de dor cervical se torna duas vezes maior (CASTILLO et al., 2016).

Estudos epidemiológicos em adultos sugerem uma associação entre cefaléia e DTM, visto que ambas apresentam sinais e sintomas similares (GONÇALVES et al., 2010). Além disso, enxaqueca é a dor de cabeça primária de maior prevalência no grupo de pacientes com DTM, sendo encontradas em $70 \%$ a $85 \%$ dos pacientes (FRANCO et al., 2011). Esta condição foi identificada em $45,5 \%$ dos participantes, mantendo a prevalência no sexo feminino, com razão de prevalência de $70 \%$ mais chances de terem o sintoma associado à DTM, confirmando a propensão das mulheres, entre 20 e 50 anos, a exibirem este sintoma (FRANCO et al., 2011). Sugere-se que prolongados impulsos nociceptivos envolvendo o núcleo caudado do trigêmeo, provenientes dos músculos da região da cabeça, além da presença de mediadores inflamatórios comuns às lesões da ATM, seriam responsáveis pela elevação da sensibilidade dolorosa e pela diminuição do limiar de dor local, cursando com sensibilização central, posteriormente contribuindo para cronificação da dor e da cefaleia (LOPES; CAMPOS; NASCIMENTO, 2010).

Ao se avaliar o comportamento da postura craniocervical no plano frontal, foi observado que inclinação/ rotação à direita ocorreu em 21 dos 47 indivíduos com DTM, assim como a inclinação/rotação à esquerda foi observada em 22 indivíduos. Quanto ao ângulo craniocervical no plano sagital entre tragus-C7 e horizontal, foram observados 19 casos de flexão craniocervical e 28 casos de extensão. Sabe-se que a cabeça possui tendência à inclinação, flexão e rotação para o lado sintomático. Isso ocorre pela hiperatividade dos músculos da mastigação reflete na musculatura cervical, determinando assim a contração dos músculos responsáveis pela elevação e protrusão dos ombros (CASTILLO et al., 2016; URITANI et al., 2014).

Outros autores relatam que a hiperatividade dos músculos mastigatórios corresponde a grande parte da etiologia das DTMs, visto que, encontraram relação entre este sinal e a postura corporal, onde os desvios estão localizados principalmente no tronco superior (URITANI et al., 2014), hiperlordose cervical ou retificação (AN et al., 2015). O aumento da lordose cervical, que também foi um achado comum neste estudo (Tabela 1), é um dos sinais mais comumente encontrados nas avaliações de postura em indivíduos com hiperatividade dos músculos da mastigação (CASTILLO et al., 2016; NOTA et al., 2017).

Os resultados deste estudo no que se refere a posição da cabeça estão de acordo com outros autores que afirmam maior prevalência das alterações posturais em portadores de DTM (BIASOTTO-GONZALEZ et al., 2008; CORRÊA et al., 2011, PASINATO; CORRÊA; SOUZA, 2009; URITANI et al., 2014). A posição anterior da cabeça 
interfere no posicionamento e funcionamento mandibular, levando a uma crescente tensão na musculatura mastigatória e, possivelmente, DTM. Essa posição leva à hiperextensão da cabeça sobre o pescoço, quando o paciente corrige para as necessidades visuais, flexão da cervical baixa sobre o tórax e posteriorização da mandíbula. Esses fatores podem levar à dor e à disfunção na cabeça e pescoço (BALDINI et al., 2016).

Diferente dos achados do atual estudo, outros autores relatam não encontrar diferenças na postura craniocervical entre indivíduos com e sem DTM. Sugerem que as alterações posturais estejam mais relacionadas à ocorrência de distúrbios craniocervicais, ou que os aspectos psicossociais podem ter influências sobre os principais sintomas das desordens e do quadro álgico, não sendo possível determinar se os desvios posturais são a causa ou o resultado da desordem (ARMIJO-OLIVO et al., 2011; CÂMARA-SOUZA et al., 2018; MATHEUS et al., 2009; SAITO; AKASHI; SACCO, 2009).

Comparando as posturas craniocervicais com as classes de oclusão dentaria demonstrados na tabela 3 , pôde-se constatar que estes dados estão de acordo com a literatura que afirma ter a posteriorização da cabeça maior prevalência na subclasse Ila (BIASOTTO-GONZALEZ et al., 2008). Com base nesses resultados, não é possível traçar nenhum parâmetro de tendência, mas apenas constatar que o comportamento da associação entre A2 e classe de Angle não tem um padrão de crescimento.

Quanto ao A3, foi verificada uma razão de prevalência de $43 \%$ de anteriorização da cabeça entre a classe lla e a classe I, $30 \%$ entre a classe IIb e a classe I e $22 \%$ entre classe III e classe I. Estes dados concordam com outros autores que citam que a classe II de maloclusão obteve maior prevalência para anteriorização da cabeça (DEDA et al., 2012).

Entre classe de oclusão dentária e postura da coluna cervical, pôde-se constatar maior razão de prevalência para anteriorização, mesmo para os indivíduos em normo-oclusão. Para os demais cruzamentos, não foi observada associação positiva, concordando com outros autores (ROSA et al., 2008). Ainda assim, outros pesquisadores encontraram postura normal de cabeça nos indivíduos classe I (GADOTTI; BERZIN; BIASOTTO-GONZALEZ, 2005).

Observa-se que a relação molar parece desempenhar um importante papel nessa ligação entre postura, cabeça e oclusão, como observado no presente estudo através da biofotogrametria. Ao contrário desses achados, outros estudos, realizados mediante avaliação visual da postura ou se admitindo outros ângulos de parâmetros craniocervicais, não encontraram relação estatística significativa entre relação dentária sagital e alterações na coluna vertebral (BIASOTTO-GONZALEZ et al., 2012; DEDA et al., 2012; MANFREDINI, et al., 2012).

A maior parte do peso do crânio descansa na região anterior da coluna cervical e nas ATM, assim sendo, a posição ortostática da relação crâniocervical é mantida por um complexo mecanismo muscular envolvendo músculos da cabeça, pescoço e escápula (CASTILLO et al., 2016; DELJO et al., 2012). A postura anterior da cabeça, achado mais prevalente neste estudo, pode levar a uma sequência de alterações no sistema crâniocervical e mandibular, como posição mais posterior da cabeça da mandíbula, compressão retrodiscal; redução do espaço funcional atlanto-occipital; aumento dos contatos dentários posteriores; desequilíbrio dos músculos cervicais e mastigatórios; e, conseqüentemente, mudança das funções estomatognáticas. Explicações para os desvios posturais incluem compensação neuromuscular para equilíbrio corporal na função trigeminal, elo neurofisiológico entre o sistema estomatognático e outros músculos do corpo (MARZ et al., 2017).

As limitações do estudo referem-se à fidedignidade no preenchimento dos questionários por parte dos participantes, a subjetividade da percepção de dor à palpação, o posicionamento estático na avaliação postural e o número da amostra reduzido. Futuros estudos necessitam de maiores parâmetros para compreender as condições multifatoriais entre as variáveis e instrumentos mais sensíveis para avaliação da dor, função muscular orofacial e cervical, severidade dos sinais e sintomas assim como, avaliação dinâmica ou global da postura corporal.

\section{CONCLUSÃO}

Com base nos resultados deste estudo, pode-se concluir que as alterações da postura craniocervical no plano frontal apresentaram associação com DTM para todos os casos e no plano sagital a associação foi positiva nos casos de classe IIb e III de Angle, assim como a anteriorização da cabeça apresentou associação com as classes II e III de Angle.

O presente estudo vem contribuir para a compreensão das associações entre as variáveis estudadas e somar-se aos conteúdos existentes na literatura. Ainda assim, estudos futuros devem incluir outros instrumentos mais sensíveis à análises das relações entre perfil postural e DTM em populações mais específicas.

\section{REFERÊNCIAS}

AN, J. S. et al. Influence of temporomandibular joint disc displacement on craniocervical posture and hyoid bone position. Am. J. Orthod. Dentofacial. Orthop, United States, v.1, n.147, p.72-9, Jan 2015.

ANGLE, E.H. Classification of malocclusion. Dent. Cosmos, Philadelphia, v.41, n.3, p.248-264, Mar. 1899.

ARMIJO-OLIVO, S. et al. Head and cervical posture in patients with temporomandibular disorders. J. Orofac. Pain., Carol Stream, v.25, n.3, p.199-209, Summer 2011.

BALDINI, A. et al. Mandibular position influence on pilots' postural balance analyzed under dynamic conditions. Cranio, England, v.6, n.35, p.367-371, Nov, 2016.

BARROS, A.J.; HIRAKATA, V. N. Alternatives for logistic regression in cross-sectional studies: an empirical comparison of models that directly 
estimate the prevalence ratio. BMC Med. Res. Methodol., England, v.3, n.21, p.1-13, Oct 2003.

BIASOTTO-GONZALEZ, D. A. et al. Análise comparativa entre dois ângulos cervicais com a oclusão em crianças com e sem DTM. Rev. CEFAC, São Paulo, v.14, n.6, p.1146-1152, dec. 2012

BIASOTTO-GONZALEZ, D. A. et al. Correlação entre disfunção temporomandibular, postura e qualidade de vida. Rev. Bras. Crescimento Desenvolv. Hum., São Paulo, v. 18, n. 1, p.79-86,abr. 2008

BOLZAN, G. P. et al. Tipo facial e postura de cabeça de crianças respiradoras nasais e orais. J. Soc. Bras. Fonoaudiol., São Paulo, v.23, n.4, p.315-320, dez. 2011.

BRANCO, R. S. et al. Freqüência de relatos de parafunções nos subgrupos diagnósticos de DTM de acordo com os critérios diagnósticos para pesquisa em disfunções temporomandibulares (RDC/TMD). Rev. Dent. Press Ortodon. Ortop. Facial, Maringá, v.13, n.2, p.61-69, abr. 2008.

CÂMARA-SOUZA, M. B. et al. Cervical posture analysis in dental students and its correlation with temporomandibular disorder. Cranio, England, v.2, n.36, p.85-90, Mar. 2018

CASTILLO, D. B. et al . Clinical study on head and jaw position of patients with muscle temporomandibular disorder. Rev. dor, São Paulo, v.17, n.2, p.88-92, June 2016.

COLARES, V.; FRANCA, C.; GONZALEZ, E. Condutas de saúde entre universitários: diferenças entre gêneros. Cad. Saúde Pública, Rio de Janeiro, v. 25, n. 3, p.00-00, mar. 2009.

CORRÊA, E.G. et al. Disfunção têmporo-mandibular e avaliação postural: uma abordagem interdisciplinar. Revista Eletrônica Saúde: Pesquisa e Reflexões, São Paulo, v.1, n.1, 2011.

DEDA, M. R. C. et al. Postura de cabeça nas deformidades dentofaciais classe II e classe III. Rev. CEFAC, São Paulo, v. 14, n. 2, p.274-280, abr. 2012.

DELJO, E. et al. Correlation analysis of the hyoid bone position in relation to the cranial base, mandible and cervical parto of vertebra with particular reference to bimaxillary relations / teleroentgenogram analysis. Acta Inform. Med., Bosnia and Herzegovina, v.1, n.20, p.2531, Mar 2012.

FRANCO, A. L. et al. Sensibilidade dolorosa à palpação em pacientes com disfunção temporomandibular crônica. Rev. Cubana Estomatol., Ciudad de La Habana, v. 48, n. 4, p.352-362, dez. 2011.

GADOTTI, I.C.; BERZIN, F.; BIASOTTO-GONZALEZ, D.A. Preliminary rapport on head posture and muscle activity in subjects with class I and II. J. Oral Rehabil., Oxford, v.32, n.11, p.784-789, Nov. 2005.

GIANNAKOPOULOS, N. N. et al. Anxiety and depression in patients with chronic temporomandibular pain and in controls. J Dent., Bristol, v.38, n.5, p.369-376, May 2010.

GONCALVES, D.A. et al. Headache and symptoms of temporomandibular disorder: an epidemiological study. Headache, St. Louis, v.50, n.2, p.231-241, Feb. 2010.
GREENACRE, M. Correspondence analysis in practice. Interdisciplinary statistics. 2. ed. Boca Raton, FL: Chapman \& Hall, 2007.

GUARDA-NARDINI, L. et al. Age-related differences in temporomandibular disorder diagnoses. Cranio, Chattanooga, v. 30, n.2, p.103-109, Apr. 2012.

KARIBE, H. et al. Comparison of subjective symptoms of temporomandibular disorders in young patients by age and gender. Cranio, Chattanooga, v.30, n.2, p.114-120, Apr. 2012.

LEMOS, G.A. et al. Correlação entre sinais e sintomas da Disfunção Temporomandibular (DTM) e severidade da má oclusão. Rev. Odontol. UNESP; São Paulo, v.3, n.44, p.175-180, maio/jun. 2015

LOPES, P.R.R.; CAMPOS, P.S.F.; NASCIMENTO, R.J.M. Dor e inflamação nas disfunções temporomandibulares: revisão de literatura dos últimos quatro anos. R. Ci. med. biol., Salvador, v.10, n.3, p.317-325, set./dez. 2011.

MANFREDINI, D. et al. Correlation of RDC/TMD axis I diagnoses and axis II pain-related disability: a multicenter study. Clin. Oral Investig., Berlin, v.15, n.5, p.749-756, Oct. 2011.

MANFREDINI, D. et al. Dentalocclusion,bodyposture and temporomandibular disorders: where we are now and wherewe are heading for. Oral Rehabil., Oxford, v.39, n.6, p.463-471, June 2012.

MANFREDINI, D.; LOBEZOO, F. Relationship between bruxism and temporomandibular disorders: a systematic review of literature from 1998 to 2008. Oral Surg. Oral Med. Oral Pathol. Oral Radiol. Endod., United States, v.6, n.109, p.26-50, June 2010.

MARZ, K. et al. Can different occlusal positions instantaneously impact spine and body posture? J. Orofac. Orthop., Germany, v.3, n.78, p.221231, Dec. 2017

MATHEUS, R.A. et al. The relationship between temporomandibular dysfunction and head and cervical posture. J. Appl. Oral Sci., Bauru, v.17, n.3, p.204-208, June 2009.

MONTEIRO, W. et al. Effectiveness of global postural reeducation in the treatment of temporomandibular disorder: Case report. J. Bodyw. Mov. Ther., New York, v.1, n.17, p.53-8, June 2013.

NOTA, A. et al. Postural stability in subjects with temporomandibular disorders and healthy controls: A comparative assessment. J. Electromyogr. Kinesiol., New York, v.37, p.21-24, Dec, 2017

PASINATO, F.; CORRÊA, E. C. R.; SOUZA, J. A. Avaliação fotogramétrica da postura da cabeça e coluna cervical de indivíduos com disfunção temporomandibular. Ter. man., Londrina, v.7, n.29, p.47-53, jan./fev. 2009.

PETERSEN, M. R.; DEDDENS, J.A. A comparison of two methods for estimating prevalence ratios. BMC Med. Res. Methodol., England, v.28, p.8-9, Feb.2008

RAINE, S.; TWOMEY, L. T. Head and shoulder posture variations in 160 asymptomatic women and men. Arch. Phys Med. Rehabil., United States, v.11, n.78, p.1215-23, Nov. 1997

RIES, L.G.K.; BERZIN, F. Analysis of the postural stability in individuals with or without signs and symptoms of temporomandibular disorder. Braz. oral res., São Paulo, v. 22, n.4, p.378-83, Dec. 2008. 
ROCHA, T. et al. Subjects with temporomandibular joint disc displacement do not feature any peculiar changes in body posture. J. Oral Rehabil., England, v.2, n.44, p.81-88, Feb. 2017.

ROSA, L. P. et al. Avaliação da postura corporal associada às maloclusões de classe II e classe III. Rev Odonto Ciênc. Porto Alegre, v.23, n.1, p.274280, Apr. 2008
SAITO, E.T.; AKASHI, P.M.H.; SACCO, I.C.N. Global body posture evaluation in patients with temporomandibular joint disorder. Clinics, São Paulo, v.64, n.1, p.35-39, Jan. 2009

URITANI, D. et al. Characteristics of Upper Quadrant Posture of Young Women with Temporomandibular Disorders. J. Phys. Ther. Sci., Japan, n.9, v.26, p.1469-1472, 2014.

Submetido em: 02/08/2020

Aceito em: $16 / 04 / 2020$ 